\title{
Short-term Load Forecasting of Local Power Grid Based on Support Vector Machine
}

\author{
Jing Hua ${ }^{1}$, Wei Xiong ${ }^{1}$ and Yanping Zhou ${ }^{2}$ \\ ${ }^{1}$ College of Engineering, Honghe College, Mengzi, Yunnan, 610000; \\ ${ }^{2}$ Kunming Power Supply Bureau, Kunming, Yunnan, 650051
}

\begin{abstract}
Short term load forecasting is an important basic work for power system planning and scheduling, short term load forecasting method based on SVM is adopted in this paper, through analyze the factors that influence the load forecasting and normalize the factors which influence the load forecasting. Using the historical load data of a city in Yunnan as the training data, the results show that the method can improve the prediction accuracy to a certain extent.
\end{abstract}

Keywords: Short term load forecasting; SVM; Prediction accuracy; Normalize.

\section{基于支持向量机的地方电网短期负荷预测}

\author{
华静1 熊伟1 周艳萍 ${ }^{2}$
}

（1. 红河学院 工学院 云南 蒙自 610000；2. 昆明供电局 云南 昆明 650051）

摘要: 短期负荷预测是电力系统制定发电计划和进行经济调度的重要基础性工作，本文采用了基于SVM的短期负荷预 测方法, 通过分析影响负荷预测的因素, 将对负荷预测影响较大的因素进行了归一化处理。利用云南某市的历史负荷数据作 为训练数据, 得到的结果表明该方法可在一定程度上提高预测精度。

关键词: 短期负荷预测; SVM; 预测精度; 归一化。

\section{1. 引言}

负荷预测是电力系统一项重要的基础性工作，是系统进行经济调度、实时控制，制定运行计划和发展 规划的前提。其中，对周、日的负荷预测称之为短期负荷预测，近些年来，随着我国电力市场的建立、发 展和不断完善, 短期负荷预测发挥的作用也日益显现, 准确的短期电力负荷预测是安排机组启停; 风、光、 水、火电协调; 联络线交换功率; 负荷经济分配; 水库调度以及设备检修等计划的前提和基础，可以提高 电力企业的经济和社会效益 $1 、 2$ 。

当前负荷预测的方法大致可分为经典的数学统计方法和基于人工智能的方法, 一些文献将神经网络 [3]、灰色系统理论 [4]、遗传算法 [5]、蚁群算法 [6 ] 等用于负荷预测中, 取得了一定成功。神经网络模 型的原始数据必须以精准为前提, 但在实际进行预测时, 由于统计存在差异, 使得统计数据和实际值有一 定差别, 导致神经网络预测模型的预测数据不理想。SVM (support vector Machine) 是针对分类和回归问 题的统计学习理论, 与神经网络采用经验风险最小化原则不同, SVM是一类基于结构风险最小化原则的新 型机器学习方法。其基本思想是通过用内积函数定义的非线性变换将输入空间变换到一个高维空间, 在这 个高维空间中寻求输入变量和输出变量之间的非线性关系的精确描述。

本文以云南某市电网的实际负荷进行预测仿真和测试, 证实所提出的模型能获得满意的预测精度, 并 将该模型运用到该地其他地区的负荷预测中。 


\section{2. 基于 SVM 的负荷预测}

\subsection{SVM原理}

对于给定的数据集, $T=\left\{x_{i}, y_{i}\right\}$,

式中 $x_{i}$ 为输入向量、 $y_{i}$ 为望值。

$f(x)=\omega^{T} x+b$

为估计函数, $x$ 为从输入空间到高维特征空间的线性映射, $\omega, b$ 可通过以下最下化式得到:

$$
\operatorname{Min} \frac{1}{2} \omega^{T} \omega+C \frac{1}{n} \sum_{i=1}^{n} L_{\varepsilon}\left(y_{i}, f\left(x_{i}\right)\right)
$$

式中 $L$ 为损失函数, 在本文中采用的是 $\varepsilon$ 不敏感损失函数, 其表达式为:

$$
L_{\varepsilon}(y, f(x))=\left\{\begin{array}{c}
0, \text { 如果 }|y-f(x) \leq \varepsilon| \\
|y-f(x)|
\end{array}\right.
$$

代入上式, 可得:

$$
\operatorname{Min} \frac{1}{2} \omega^{T} \omega+C \sum_{i=1}^{n}\left(\xi_{i}+\stackrel{*}{\xi}_{i}\right)
$$

约束条件为

$$
\left\{\begin{array}{c}
\omega x_{i}+b_{i}-y_{i} \leq \varepsilon+\stackrel{*}{\xi}_{i} \\
-\omega x_{i}-b_{i}+y_{i} \leq \varepsilon+\xi_{i} \\
\xi_{i}, \xi_{i}^{*} \geq 0
\end{array}\right\}
$$

在式 (5)、(6) 中, C为常数, 称为惩罚因子, $\xi_{i}, \xi_{i}^{*}$ 为引入的松驰变量, 通过构造拉格朗日函数可得其 对偶函数为:

$$
\min \frac{1}{2} \sum_{i=1}^{l} \sum_{j=1}^{l}\left(\alpha_{i}-\alpha_{i}^{*}\right)\left(\alpha_{j}-\alpha_{j}^{*}\right)\left(x_{i}^{T} * x_{j}\right)+\sum_{i=1}^{l} y_{i}\left(\alpha_{i}-\alpha_{i}^{*}\right)-\varepsilon \sum_{i=1}^{l}\left(\alpha_{i}+\alpha_{i}^{*}\right)
$$

约束条件为: $\sum_{i=1}^{n}\left(\alpha_{i}-\alpha_{i}^{*}\right)=0, \alpha_{i}, \alpha_{i} \in[0, c]$

根据Karush-Kuhn-Tucker定理, 可求得 $\alpha_{i},{ }^{*} \alpha_{i}, b$, 最终可得到SVM的回归函数为:

$$
f(x)=\sum_{i=1}^{n}\left(\alpha_{i}-\alpha_{*}^{i}\right) \cdot k\left(x_{i}, x\right)+b
$$

式 (9) 中 $k\left(x_{i}, x\right)$ 为特征空间的一个内积, 称之为核函数, 任何对称函数只要满足Mercer定理, 均可作 为核函数。利用核函数后, 就不再需要知道映射具体形式, 不需要直接处理高维特征空间, 只用给出核函 
数即可。常用的核函数有多项式核函数、RBF核函数、sigmoid核函数。

\section{2 负荷输入及其量化处理}

\subsection{1 影响短期负荷预测精度的因素}

(1) 经济因素

经济环境的好坏和经济发展状况对负荷预测是有重要影响的。一般来说, 经济发展比较好的情况下, 负荷水平就提升的比较快; 反之，负荷水平就会下降;

(2) 气象因素

影响负荷的气象因素很多, 主要包括温度、湿度、日照强度、降雨等, 在这些因素中温度对负荷影响 最大, 考虑电网当前获得实际气象数据来源情况, 在模型输入中只计及温度, 其余均忽略, 再加上气象预 报本身的不准确，会造成双重误差。

(3) 样本因素

影响短期电力负荷预测的样本因素包括样本数量、样本质量和样本范围。在进行短期电力负荷预测时, 不能仅仅考虑时间、历史数据因素，还应该综合考虑影响负荷的各种因素，同时对各个因素进行定量和定 性的分析，进而选择最佳样本，使预测更加准确。

(4) 日期类型

根据该市实际电网的负荷特点，日期分类为工作日(周一～周日)、一般休息日 (周六、周日)、节假日 (元旦、春节、五一、中秋、十一等)，不同的日期类型，负荷出现的大小也不一样。

\subsection{2 负荷样本处理}

（1）负荷数据的归一化处理

在原始数据中找出最大值, 再用所有数据去除, 即可将原始数据均变为小于 1 的值, 方便在Matlab软 件里运行;

$$
y=\frac{x}{x_{\max }}
$$

(2) 天气和温度特征的归一化

负荷波动受到温度变化的影响, 当温度变化在一定范围内时, 负荷也在一定的范围内变化波动。文中 将温度的归一化处理与负荷的归一化处理相结合。

云南四季如春，但也有 “一雨变成秋” 的说法，进行负荷预测的地区地处滇中，因此，根据其气候特 点，将春、夏、秋分为一类，冬天为一类，分别进行量化处理，见表 $1 、 2$

(3) 日期量化处理

文中将日期分为三个类型：工作日(周一～周日)、一般休息日(周六、周日)、节假日 (元旦、春节、 五一、中秋、十一等)。 
表1 春夏秋天气量化处理

\begin{tabular}{ccccccccccc}
\hline 天气状况 & 晴 & 雾 & 多云 & 阴 & 小雨 & 中雨 & 大雨 & 阵雨 & 雷雨 & 暴雨 \\
\hline 量化值 & 1 & 0.9 & 0.8 & 0.7 & 0.6 & 0.5 & 0.4 & 0.3 & 0.2 & 0.1 \\
\hline
\end{tabular}

表2 冬季天气量化处理

\begin{tabular}{lllllllll}
\hline 天气状况 & 晴 & 多云 & 阴 & 小雨 & 中雨 & 暴雨 & 雨雪 & 小雪 \\
\hline 量化值 & 1 & 0.9 & 0.8 & 0.7 & 0.6 & 0.5 & 0.4 & 0.2 \\
\hline
\end{tabular}

\section{3 基于 SVM 的负荷预测步骤}

用 SVM 预测功率的步骤如下所示:

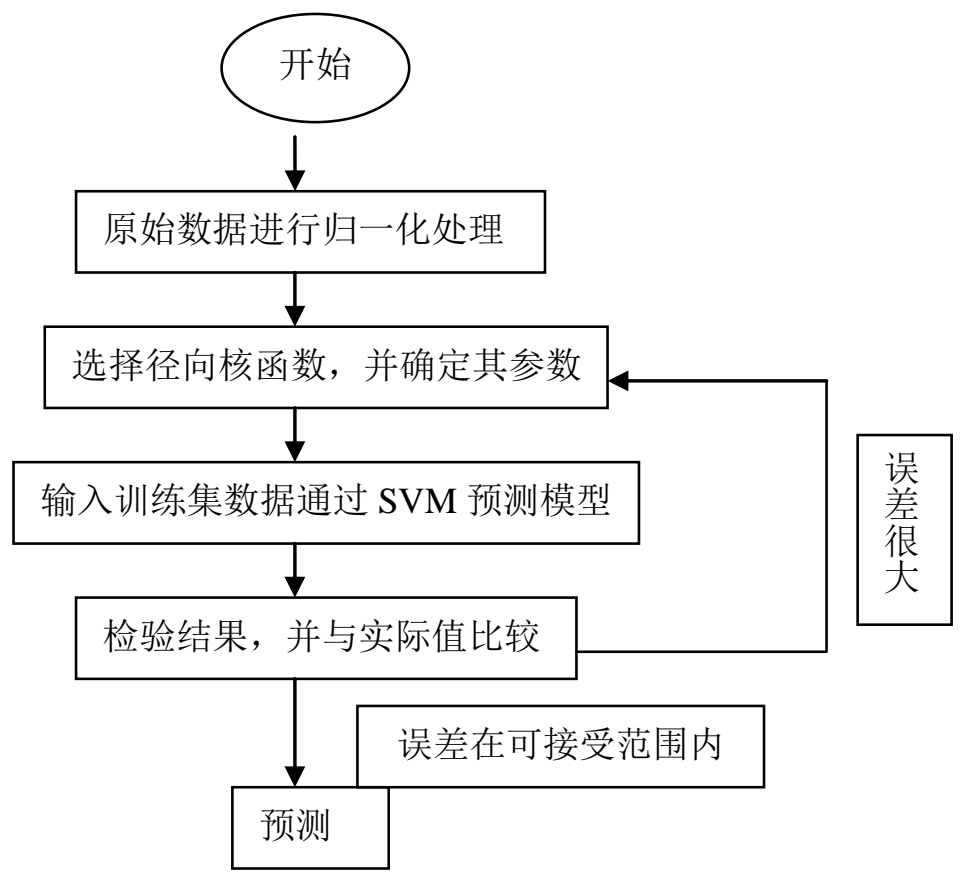

图1 SVM预测功率步骤

（1）对历史数据进行归一化处理;

（2）输入训练数据，根据所编的程序运行，建立预测模型;

(3) 降训练数据的预测结果与实际历史数据比较, 如果误差结果符合预想, 既可以进行预测, 如果偏差 较大, 则返回步骤 2 重新修改参数值, 再次进行预测。

\section{3. 算例分析}

运用文中所讲的SVM预测方法，利用该市2015年1月5日、6日的历史负荷数据作为训练样本，对7日全 天的负荷进行预测，期预测结果如表3所示: 
表3 云南某市1月7日负荷预测结果统计表

\begin{tabular}{|c|c|c|c|}
\hline 时刻/h & 实际负荷 / (MW) & 预测负荷 / (MW) & 相对误差/\% \\
\hline $0: 00$ & 3122.445 & 3087.732 & 1. 111 \\
\hline $1: 00$ & 2849.984 & 2783. 432 & 2. 335 \\
\hline $2: 00$ & 2685.978 & 2643. 236 & 1.591 \\
\hline $3: 00$ & 2602.688 & 2514.977 & 1. 337 \\
\hline $4: 00$ & 2500.071 & 2459.133 & 1. 663 \\
\hline $5: 00$ & 2498.422 & 2512. 488 & -0.563 \\
\hline $6: 00$ & 2504.458 & 2473.603 & 1. 232 \\
\hline $7: 00$ & 2603. 291 & 2554.167 & 1. 887 \\
\hline $8: 00$ & 2814.32 & 2840.155 & -0.918 \\
\hline $9: 00$ & 3244.623 & 3201.437 & 1. 331 \\
\hline $10: 00$ & 3539.533 & 3488.458 & 1. 443 \\
\hline $11: 00$ & 3610.608 & 3541.176 & 1.923 \\
\hline $12: 00$ & 3463.896 & 3510.659 & -1.135 \\
\hline $13: 00$ & 3326.453 & 3296.548 & 0.899 \\
\hline $14: 00$ & 3265. 075 & 3214.575 & 1.562 \\
\hline $15: 00$ & 3220.415 & 3180.417 & 1. 242 \\
\hline $16: 00$ & 3234.669 & 3229.008 & 0.175 \\
\hline $17: 00$ & 3426.332 & 3394.227 & 0.937 \\
\hline $18: 00$ & 3486.544 & 3450.563 & 1. 032 \\
\hline $19: 00$ & 3488.258 & 3458.015 & 0.867 \\
\hline $20: 00$ & 3563.685 & 3515.932 & 1. 134 \\
\hline $21: 00$ & 3615.204 & 3575.362 & 1. 091 \\
\hline $22: 00$ & 3534.989 & 3569.031 & -0.963 \\
\hline $23: 00$ & 3201.297 & 3101.297 & 1. 313 \\
\hline \multicolumn{3}{|c|}{ 平均相对误差 } & 0.939 \\
\hline
\end{tabular}

从表三可以看出。采用SVM预测模型预测准确性较好, 平均相对误差仅为 0.939 , 精度较高, 预测结果 可靠, 应用在实际中具有更强的说服力。

\section{4. 结语}

(1) 通过利用SVM预测模型对地方负荷进行预测仿真, 取得了较高的精度, 证明该预测方法可满足实际 调度负荷预测需求，具有一定的应用价值;

(2) 文中对气象状况和日期类型进行了归一化处理, 既保证了训练数据的有效性和可靠性, 又极大地 提高了输入量在Matlab中的运行速度和精度。 


\section{5. 致谢}

基金项目：云南省教育厅科学研究基金资助项目 (2015Y453)

\section{Acknowledgement}

Fund Project: Yunnan Provincial Department of Education Science Research Fund Project (2015Y453)

\section{参考文献:}

[1] 康重庆, 夏清, 张伯明. 电力系统负荷预测综述与发展方向的探讨 [J] 电力系统自动化, 2004, 28 (17)：1-11

[2] 李永斌. 短期电力负荷预测模型的建立与应用 $[\mathrm{J}]$ 计算机仿真，2011，28(10)：316-319

[3] 陆宁, 周建忠, 何耀耀. 粒子群优化的神经网络模型在短期负荷预测中的应用 [ J J . 电力系统保护与控制, $2010,38(12): 65-68$

[4] 李家科, 李亚娇, 李怀恩, 等. 非点源污染负荷预测的多变量灰色神经网络模型 $[\mathrm{J}]$. 西本农林科技大学学报(自 然科学版), 2011, 39(3): 229-234

[5] 张勇, 杨云. 改进 GA-BPNN 在短期负荷预测中的应用 [J] . 计算机工程与设计, 2008, 29 (7)：797-1 799, 1837.

[6] 王晶, 刘博, 冯艳红. 蚁群神经网络在短期负荷预测的应用 [J] . 北京信息科技大学学报, 2010, 25 (2)：128-131

[7] 孙薇, 刘默涵. 基于改进最小二乘支持向量机的短期负荷预测 $[\mathrm{J}]$. 电力科学与工程, 2015,31(12)：16-21

\section{References:}

[1] Kang Chongqing, Xia Qing, Zhang Boming. Summary of power system load forecasting and discussion on development direction [J] Automation of Electric Power Systems, 2004, 28 (17): $1-11$

[2] Li Yongbin. Establishment and application of short-term power load forecasting model [J] Computer Simulation, 2011,28 (10): 316-319

[3] Lu Ning, Zhou Jianzhong, He Yaoyao. Application of Neural Network Model Based on Particle Swarm Optimization in Short-term Load Forecasting [J]. Power System Protection and Control, 2010, 38 (12): 65-68

[4] Li Jiake, Li Yajiao, Li Huaien, et al. Multivariable gray neural network model for non-point source pollution load forecasting [J]. Journal of West China Agricultural University (Natural Science Edition), 2011,39 (3): 229-234

[5] Zhang Yong, Yang Yun. Application of improved GA-BPNN in short-term load forecasting [J]. Computer Engineering and Design, 2008, 29 (7): 797-1 799,1 837.

[6] Wang Jing, Liu Bo, Feng Yanhong. Application of ant colony neural network in short-term load forecasting [J]. Journal of Beijing Information Technology University, 2010,25 (2): 128-131

[7] Sun Wei, Liu Mohan. Short-term load forecasting based on improved least squares support vector machine [J]. Electric Power Science and Engineering, 2015,31 (12): 16-21 